\title{
REFERENCES
}

1. S. Bergman, The kernel function and conformal mapping, Mathematical Surveys, no. 5, American Mathematical Society, New York, 1950.

2. S. Bochner and W. T. Martin, Several complex variables, Princeton University Press, 1948.

3. G. Scheffers, Math. Zeit. vol. 2, p. 181.

[1] and [2] contain extensive bibilographies.

WASHINGTON UNIVERSITY

\section{A PROPERTY OF QUASI-CONFORMAL MAPPING}

\author{
H. L. ROYDEN
}

Let $W_{1}$ and $W_{2}$ be two open Riemann surfaces such that there is a quasi-conformal [1] mapping $h$ of $W_{1}$ onto $W_{2}$. Then it is known [2] that either both $W_{1}$ and $W_{2}$ have Green's function or else neither has, i.e., quasi-conformal mapping preserves the class $O_{G}$ of those surfaces which have no Green's function. From this one is led to the conjecture that quasi-conformal mapping preserves the classes $O_{H B}$ and $O_{H D}$ of surfaces which have no bounded harmonic function or harmonic functions with a finite Dirichlet integral, respectively. In the present paper we shall show that the ring $H D$ of bounded harmonic functions with a finite Dirichlet integral is the same for both $W_{1}$ and $W_{2}$. This has as a consequence not only the result of Pfluger on the preservation of the class $O_{G}$ but also the preservation of the class $O_{H D}$ under quasi-conformal mapping.

Whether or not $O_{H B}$ is preserved under quasi-conformal mapping is an open question. Another interesting open question is whether or not two topologically equivalent surfaces which have the same ring $H B D$ defined on them admit of a quasi-conformal mapping from one to the other.

1. Some rings of functions. Let $B D=B D_{i}$ be the ring of all piecewise smooth functions defined on the Riemann surface $W=W_{i}$ which are bounded and have a finite Dirichlet integral. A topology is introduced in $B D$ by defining

$$
f_{,} \rightarrow f
$$

if $\left|f_{\nu}\right|$ is uniformly bounded, $f$ converges to $f$ uniformly on every

Received by the editors July 27, 1953. 
compact subset of $W$, and $D\left[f_{\nu}-f\right]$ converges to zero, where $D[]$ denotes the Dirichlet integral:

$$
D[f]=\iint d f * d \bar{f} .
$$

A subset $S$ of $B D$ is then called closed if it contains all limits of sequences from $S$.

We let $K$ be the ideal consisting of all those functions in $B D$ which vanish outside some compact set and denote by $\bar{K}$ those functions which are limits (in $B D$ ) of sequences from $K$. It is shown in [3] that $\bar{K}$ is closed and that every function $f \in B D$ has a unique decomposition

$$
f=f_{K}+u
$$

where $f_{K} \in \bar{K}$ and $u \in H B D$, the space of harmonic functions in $B D$ (with the convention that the constants belong to $H B D$ only if $W$ is hyperbolic). As a vector space $H B D$ is isomorphic to the quotient space $B D / \bar{K}$ and we use this isomorphism to define [4] a multiplication in $H B D$.

If we let $\pi$ be the homomorphism which takes $f$ into the harmonic part $u$ of its decomposition (1), then $\pi$ is a ring homomorphism by the definition of multiplication in $H B D$. Moreover, $\pi$ is continuous.

2. A class of linear functionals. Let $L$ consist of those continuous linear functionals on $B D$ which annihilate $\bar{K}$. Then $L$ is a total set of linear functionals for the subspace $H B D$. For, if $u \in H B D$ and $u$ is not constant, then

$$
l(f)=\iint d f * d \bar{u}
$$

is continuous on $B D$ and annihilates functions in $\bar{K}$. But

$$
l(u)=D[u]>0 .
$$

Also there is by Theorem 3 of [3] a linear functional $l \in L$ such that

$$
l[1] \neq 0 \text {. }
$$

Now if $l \in L$, then

$$
l[f]=l[(1-\pi) f]+l[\pi f]=l(\pi f)
$$

since $(1-\pi) f \in \bar{K}$.

3. Mappings induced by $h$. Let now $h$ be a quasi-conformal mapping of $W_{1}$ onto $W_{2}$. Then we let $\tau$ be the mapping of $B D_{1}$ onto $B D_{2}$ defined by 


$$
\tau f(P)=f\left[h^{-1}(P)\right]
$$

and we have

$$
\tau^{-1} f(Q)=f[h(Q)],
$$

$Q \in W_{1}$.

Moreover, $\tau$ is a homeomorphism of $B D_{1}$ onto $B D_{2}$. For if $\left|f_{\nu}\right|$ is uniformly bounded so is $\left|\tau f_{\nu}\right|$; while if $f_{\nu}$ converges to $f$ uniformly on compact subsets of $W_{1}$, then $\tau f_{\nu}$ converges to $\tau f$ uniformly on compact subsets of $W_{2}$. Also,

$$
D\left[\tau f_{\nu}-\tau f\right] \leqq k D\left[f-f_{v}\right],
$$

where $k$ is the bound on the dilation quotient of the mapping $h$. Hence $\tau$ is continuous. Similarly $\tau^{-1}$ is continuous and $\tau$ is a homeomorphism. Since $\tau$ is linear, it is also an isomorphism.

We define $\sigma$ to be the adjoint of $\tau^{-1}$, i.e., for a continuous linear functional $l$ on $B D_{1}$ we define $l \sigma$ by

$$
l \sigma[f]=l\left[\tau^{-1} f\right], \quad \quad f \in B D_{2} .
$$

Then

$$
l \sigma[\tau f]=l[f] .
$$

Since $\tau^{-1}$ takes $\bar{K}_{2}$ into $\bar{K}_{1}, \sigma$ takes $L_{1}$ into $L_{2}$.

4. The principle theorem. We first prove the following relations

$$
\begin{aligned}
& \pi_{1} \tau^{-1} \pi_{2} \tau \pi_{1}=\pi_{1}, \\
& \pi_{2} \tau \pi_{1} \tau^{-1} \pi_{2}=\pi_{2} .
\end{aligned}
$$

For any $l \in L_{1}$ and $u \in H B D_{1}$, we have

$$
l\left[\pi_{1} \tau^{-1} \pi_{2} \tau u\right]=l\left[\tau^{-1} \pi_{2} \tau u\right]
$$

by (2). By the definition of $\sigma$ we may write this as

$$
\begin{aligned}
l\left[\pi_{1} \tau^{-1} \pi_{2} \tau u\right] & =l \sigma\left[\pi_{2} \tau u\right] \\
& =l \sigma[\tau u]
\end{aligned}
$$

by (2) since $l \sigma \in L_{2}$. By (3) we have

$$
l\left[\pi_{1} \tau^{-1} \pi_{2} \tau u\right]=l[u] .
$$

Since $\pi_{1} \tau^{-1} \pi_{2} \tau u \in H B D_{1}$ and $L_{1}$ is total for $H B D_{1}$, we must have

$$
\pi_{1} \tau^{-1} \pi_{2} \tau u=u
$$

whence (5) follows. Similarly for (6).

THEOREM 1. The mapping $\pi_{2} \tau$ is a homeomorphic isomorphism of the 
ring $H B D_{1}$ onto the ring $H B D_{2}$.

Proof. The mapping $\pi_{2} \tau$ is a ring homomorphism since

$$
\pi_{2} \tau=\pi_{2} \tau \pi_{1}
$$

on $H B D_{1}$,

and $\pi_{2}, \tau$, and $\pi_{1}$ are all ring homomorphisms. Moreover, $\pi_{2} \tau$ is continuous since $\pi_{2}$ and $\tau$ are. So also is $\pi_{1} \tau^{-1}$. But by (5) and (6)

$$
\left(\pi_{2} \tau\right)^{-1}=\pi_{1} \tau^{-1}
$$

whence $\pi_{2} \tau$ is one-to-one onto and bicontinuous, proving the theorem.

Since $W \in O_{G}$ is equivalent to $H B D(W)$ empty and $W \in O_{H D}$ is equivalent to saying $H B D(W)$ has dimension less than two, we have the following corollaries:

Corollary 1. The class $O_{G}$ is preserved under quasi-conformal mapping.

CoROllaRy 2. The class $O_{H D}$ is preserved under quasi-conformal mapping.

\section{BIBLIOGRAPHY}

1. L. Ahlfors, Zur Theorie der Überlagerunsfiächen, Acta Math. vol. 65 (1935).

2. A. Pfluger, Sur un propriêté de l'application quasi conforme d'une surface de Riemann ouverte, C. R. Acad. Sci. Paris vol. 227 (1948) pp. 25-26.

3. H. L. Royden, Harmonic functions on open Riemann surfaces, Trans. Amer. Math. Soc. vol. 73 (1952) pp. 40-94.

4. - - The ideal boundary of an open Riemann surface, "Contributions to the Theory of Riemann Surfaces," Annals of Mathematics Studies, No. 30, Princeton University Press.

Stanford University 